\title{
Scaling method for the pair-density-functional theory in combination with energy functionals satisfying the virial theorem: Checking the validity via atomic-structure calculations
}

\author{
Katsuhiko Higuchi \\ Graduate School of Advanced Sciences of Matter, Hiroshima University, Higashi-Hiroshima 739-8527, Japan \\ Masahiko Higuchi \\ Department of Physics, Faculty of Science, Shinshu University, Matsumoto 390-8621, Japan
}

(Received 11 December 2012; published 20 March 2013)

\begin{abstract}
We perform atomic-structure calculations for the neutral $\mathrm{Ne}, \mathrm{Mg}$, and $\mathrm{Ar}$ atoms on the basis of the recently proposed correction method (scaling method) for the pair-density (PD) -functional theory [Phys. Rev. A 84, 044502 (2011)]. The formal features of the scaling method are that the search region of PDs is substantially extended and that the resultant variationally best PD, which can be obtained without the heavy calculation tasks, satisfies the virial theorem rigorously. To enjoy the benefit of these features, we also develop the approximate form of the kinetic energy functional. It is shown by the atomic-structure calculations that the scaling method can improve well not only various energy functionals but also the spatial profiles of the electron density and exchange-correlation hole. Especially it is found that the scaling method makes preferential modifications to the energetically effective regions of the electron density and exchange-correlation hole. These results suggest that the scaling method efficiently puts the PD close to the correct ground-state PD.
\end{abstract}

DOI: 10.1103/PhysRevA.87.032511

PACS number(s): 31.15.E-, 31.15.ve, 31.15.xt, 71.15.Mb

\section{INTRODUCTION}

The density-functional theory (DFT) [1,2] is one of useful first-principles theories that provide the ground-state properties of the condensed-matter systems. Aiming at developing the theory that guarantees to reproduce various physical quantities besides the electron density, the DFT has been generalized or extended [3-39]. Specifically, the physical quantities that are characteristic of the ground state of the system are chosen as basic variables of the theories [3-39]. Among them, the pair-density (PD) -functional theory [15-39], in which the PD is chosen as the basic variable, is considered as a promising theory that goes beyond the conventional DFT. This is because the PD includes more information about the electron correlation than the electron density.

In order to develop the PD functional theory, there are two problems that need to be overcome. In what follows, we shall denote them as (I) and (II), and explain the outlines in order.

(I) One is how to search the best PD over the whole set of $N$-representable PDs when we employ the variational principle with respect to the PD. This problem is attributed to the lack of practical knowledge about the necessary and sufficient conditions for the $N$-representability of PDs [40-56]. This problem is very important because the variational principle may possibly lead to an unphysical solution as the best PD unless we devise some practical method to avoid this problem. As such a device, we have previously proposed an effective initial scheme, in which the set of PDs that are calculated from single Slater determinants (SDs) is used as the search region of PDs [28,30,32]. As other ones, the Jastrow wave function [29] and the linear combination of the SDs [33] have also been attempted in constructing the search region of PDs. More recently, we have proposed an efficient method to extend the search region of PDs [34] by adding the scaled PDs as elements of the search region. The scaled PDs are calculated from the antisymmetric wave functions that are transformed by uniformly scaling the electron coordinates, and they are obviously $N$-representable [34]. The striking feature of this method is that it satisfies the virial theorem rigorously [34]. Although it is a preliminary work, we have shown that the PD can be corrected to be close to the ground-state one by this method [34]. Hereafter, in this paper, this method is called the scaling method [34].

(II) Another problem in developing the PD functional theory is that the approximate form of the kinetic energy (KE) is needed to be developed as a functional of the PD [35-39]. This is because the KE cannot be rigorously written by only the PD [35-39]. Concerning this problem, we have taken two strategies for developing the approximate functional. (a) One is to develop the approximate form on the basis of the coupling-constant expression for the KE functional [39]. (b) The other is to develop the approximate form by imposing on it relations and bounds that are fulfilled by the exact KE functional as restrictive conditions [33,34,39]. Along each strategy, we have begun to propose the approximate forms of the KE functional [33,34,39].

For developing the PD functional theory, we have to consider both problems, (I) and (II), simultaneously. In this paper, we employ the scaling method [problem (I)], and adopt the approximate $\mathrm{KE}$ functional which is suitable to bring out the advantageous features of the scaling method [problem (II)]. In order to check the validities of the present scheme, numerical calculations are performed for the neutral $\mathrm{Ne}, \mathrm{Mg}$, and Ar atoms systematically. We investigate not only the electron-electron interaction energy, external potential energy, and $\mathrm{KE}$ but also the electron density and exchange-correlation (xc) hole. The spatial profile of the xc hole especially seems to be interesting in a sense of the direct investigation of the PD. These results will be shown in the subsequent sections. Furthermore in this paper, the derivation of the approximate $\mathrm{KE}$ functional is given in details because it would be useful for developing other kinds of approximate forms. 
Organization of this paper is as follows. In Sec. II, the scaling method is explained with stressing its formal features. In Sec. III, we first derive the restrictive conditions on the $\mathrm{KE}$ functional, and secondly we propose the approximate $\mathrm{KE}$ functional by using these conditions. To check the validity of the scaling method combined with the proposed KE functional, numerical calculations for the neutral $\mathrm{Ne}, \mathrm{Mg}$, and $\mathrm{Ar}$ atoms are performed in Sec. IV. Finally, some concluding remarks are given in Sec. V.

\section{SCALING METHOD FOR THE PD FUNCTIONAL THEORY}

In Sec. II A, we first derive the virial theorem of the PD functional theory and show that the virial theorem can be utilized in evaluating the incompleteness of the search region of PDs. In Sec. II B, we present the concrete prescription for how to augment the incomplete search region of PDs, i.e., we shall present the scaling method. The formal feature of the scaling method is that the search region of PDs is augmented so that the corrected PD satisfies the virial theorem. It is discussed in Sec. II C.

\section{A. Incompleteness of the search region of PDs}

The PD $\gamma^{(2)}\left(\mathbf{r} \mathbf{r}^{\prime} ; \mathbf{r} \mathbf{r}^{\prime}\right)$ is defined as the diagonal elements of the second-order reduced density matrix, which is written as

$$
\begin{aligned}
\gamma^{(2)}\left(\mathbf{r r}^{\prime} ; \mathbf{r r}^{\prime}\right)= & \frac{N(N-1)}{2} \int \Psi^{*}\left(\mathbf{r}, \mathbf{r}^{\prime}, \mathbf{r}_{3}, \cdots, \mathbf{r}_{N}\right) \\
& \times \Psi\left(\mathbf{r}, \mathbf{r}^{\prime}, \mathbf{r}_{3}, \cdots, \mathbf{r}_{N}\right) d^{3} r_{3} \cdots d^{3} r_{N},
\end{aligned}
$$

where $\Psi\left(\mathbf{r}_{1}, \cdots, \mathbf{r}_{N}\right)$ is the antisymmetric wave function of the $N$-electron system. We consider the transformation of electron coordinates $\mathbf{r}_{i}(i=1, \cdots, N)$ into $\lambda^{-1} \mathbf{r}_{i}$, where $\lambda$ denotes the scaling factor and is assumed to be positive. This type of transformation is called a uniform coordinate scaling of electrons. By the uniform coordinate scaling of electrons, the wave function $\Psi\left(\mathbf{r}_{1}, \cdots, \mathbf{r}_{N}\right)$ is transformed into

$$
\Psi_{\lambda}\left(\mathbf{r}_{1}, \cdots, \mathbf{r}_{N}\right)=\lambda^{\frac{3 N}{2}} \Psi\left(\lambda \mathbf{r}_{1}, \cdots, \lambda \mathbf{r}_{N}\right),
$$

where $\lambda^{3 N / 2}$ is a normalizing constant. Using Eq. (1), the PD that is calculated from $\Psi_{\lambda}\left(\mathbf{r}_{1}, \cdots, \mathbf{r}_{N}\right)$ is given by

$$
\gamma_{\lambda}^{(2)}\left(\mathbf{r r}^{\prime} ; \mathbf{r r}^{\prime}\right)=\lambda^{6} \gamma^{(2)}\left(\lambda \mathbf{r} \lambda \mathbf{r}^{\prime} ; \lambda \mathbf{r} \lambda \mathbf{r}^{\prime}\right) \text {. }
$$

This PD is hereafter called the scaled PD. It should be noted that the scaled PD is necessarily $N$-representable.

The total energy functional of the PD functional theory is given by

$$
E\left[\gamma^{(2)}\right]=T\left[\gamma^{(2)}\right]+W\left[\gamma^{(2)}\right]+V\left[\gamma^{(2)}\right],
$$

where $T\left[\gamma^{(2)}\right], W\left[\gamma^{(2)}\right]$ and $V\left[\gamma^{(2)}\right]$ denote the functionals of the KE, electron-electron interaction energy, and external potential energy, respectively. As is shown in the previous work [19], the KE functional for the scaled PD is related to $T\left[\gamma^{(2)}\right]$ by

$$
T\left[\gamma_{\lambda}^{(2)}\right]=\lambda^{2} T\left[\gamma^{(2)}\right] .
$$

This exact relation is utilized in Sec. III as one of the restrictive conditions on the approximate form of the KE functional. In the following discussions, we assume that the approximate form of the KE functional satisfies the exact relation Eq. (5) [57].

Unlike $T\left[\gamma^{(2)}\right]$, both $W\left[\gamma^{(2)}\right]$ and $V\left[\gamma^{(2)}\right]$ are exactly expressed in terms of the PD, i.e.,

$$
\begin{gathered}
W\left[\gamma^{(2)}\right]=\frac{e^{2}}{2} \iint \frac{\gamma^{(2)}\left(\mathbf{r} \mathbf{r}^{\prime} ; \mathbf{r r}^{\prime}\right)}{\left|\mathbf{r}-\mathbf{r}^{\prime}\right|} d^{3} r d^{3} r^{\prime}, \\
V\left[\gamma^{(2)}\right]=\frac{2}{N-1} \iint v_{\mathrm{ext}}(\mathbf{r}) \gamma^{(2)}\left(\mathbf{r} \mathbf{r}^{\prime} ; \mathbf{r} \mathbf{r}^{\prime}\right) d^{3} r d^{3} r^{\prime},
\end{gathered}
$$

respectively, where $v_{\text {ext }}(\mathbf{r})$ denotes the external potential. Using Eqs. (3), (6), and (7), the electron-electron interaction and external potential energies for the scaled PD are calculated as

$$
\begin{gathered}
W\left[\gamma_{\lambda}^{(2)}\right]=\lambda W\left[\gamma^{(2)}\right], \\
V\left[\gamma_{\lambda}^{(2)}\right]=\frac{2}{N-1} \iint v_{\mathrm{ext}}\left(\frac{\mathbf{r}}{\lambda}\right) \gamma^{(2)}\left(\mathbf{r} \mathbf{r}^{\prime} ; \mathbf{r r}^{\prime}\right) d^{3} r d^{3} r^{\prime},
\end{gathered}
$$

where we suppose in the derivation of Eq. (9) that the surface integral at infinity can be neglected. If $v_{\text {ext }}(\mathbf{r})$ is the external potential energy of an isolated atomic system, i.e., $v_{\text {ext }}(\mathbf{r}) \propto$ $1 /|\mathbf{r}|$, then Eq. (9) is simply rewritten as

$$
V\left[\gamma_{\lambda}^{(2)}\right]=\lambda V\left[\gamma^{(2)}\right]
$$

Next, we consider two cases that are shown in Figs. 1(a) and 1(b). One is the case where the search region of PDs $\left(C_{1}\right)$ is identical with the set of all $N$-representable PDs $\left(C_{\text {all }}\right)$, and another case is that $C_{1}$ is a proper subset of $C_{\text {all }}$. Before considering these two cases, we summarize in advance the notations used below. We denote by $\tilde{\gamma}_{0}^{(2)}$ the PD that belongs to $C_{1}$ and gives the minimum of $E\left[\gamma^{(2)}\right]$, i.e.,

$$
\underset{\gamma^{(2)} \in C_{1}}{\operatorname{Min}} E\left[\gamma^{(2)}\right]=E\left[\tilde{\gamma}_{0}^{(2)}\right] \text {. }
$$

The scaled PD that is calculated from $\tilde{\gamma}_{0}^{(2)}$ is denoted by $\tilde{\gamma}_{0, \lambda}^{(2)}$. By changing the value of $\lambda$, the set of $\tilde{\gamma}_{0, \lambda}^{(2)}$ can be obtained. We denote the set as $C_{2}$, and call $\tilde{\gamma}_{0}^{(2)}$ the "seed PD" in this paper.

Let us consider the former case [Fig. 1(a)], namely the case of $C_{1}=C_{\text {all }}$. In this case, $C_{1}$ contains all elements of $C_{2}$ because all scaled PDs $\tilde{\gamma}_{0, \lambda}^{(2)}$ are $N$-representable. Therefore, $E\left[\tilde{\gamma}_{0, \lambda}^{(2)}\right]$ should take a minimum value at $\lambda=1$. Namely, we

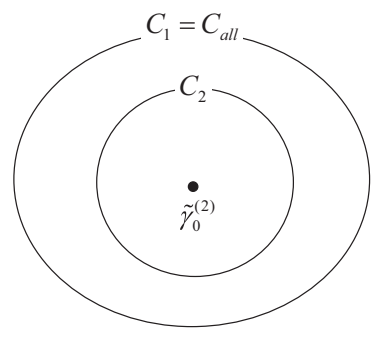

(a)

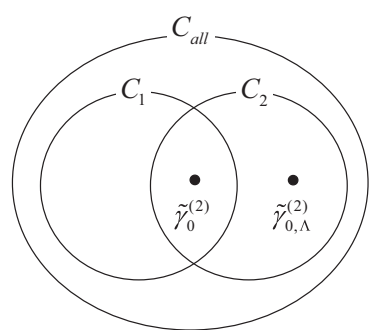

(b)
FIG. 1. Schematic view of the search region of PDs. The symbols $C_{\text {all }}, C_{1}$, and $C_{2}$ denote the set of $N$-representable PDs, the search region of PDs, and the set of scaled PDs, respectively. (a) The case where $C_{1}$ is identical with $C_{\text {all }}$. (b) The case where $C_{1}$ is a proper subset of $C_{\text {all }}$. 
have

$$
\left.\frac{d E\left[\tilde{\gamma}_{0, \lambda}^{(2)}\right]}{d \lambda}\right|_{\lambda=1}=0 .
$$

Using Eqs. (5), (8), and (9), Eq. (12) leads to the virial theorem of the PD functional theory:

$$
\begin{aligned}
& 2 T\left[\tilde{\gamma}_{0}^{(2)}\right]+W\left[\tilde{\gamma}_{0}^{(2)}\right]-\frac{2}{N-1} \int\left\{\mathbf{r} \cdot \nabla v_{\mathrm{ext}}(\mathbf{r})\right\} \\
& \quad \times\left\{\int \tilde{\gamma}_{0}^{(2)}\left(\mathbf{r r}^{\prime} ; \mathbf{r r}^{\prime}\right) d^{3} r^{\prime}\right\} d^{3} r=0,
\end{aligned}
$$

where we use the fact that $\tilde{\gamma}_{0,1}^{(2)}$ is identical with $\tilde{\gamma}_{0}^{(2)}$. If Eq. (10) is used instead of Eq. (9), then Eq. (13) is rewritten as a more familiar form of the virial theorem:

$$
2 T\left[\tilde{\gamma}_{0}^{(2)}\right]+W\left[\tilde{\gamma}_{0}^{(2)}\right]+V\left[\tilde{\gamma}_{0}^{(2)}\right]=0 .
$$

Equation (13) [or (14)] means that the virial theorem holds for the seed PD $\tilde{\gamma}_{0}^{(2)}$ in the case of $C_{1}=C_{\text {all }}$. That is to say, if the virial ratio $R_{\text {virial }}\left[\gamma^{(2)}\right]$ is defined by

$$
\begin{aligned}
& R_{\text {virial }}\left[\gamma^{(2)}\right] \\
& =\frac{W\left[\gamma^{(2)}\right]-\frac{2}{N-1} \int\left\{\mathbf{r} \cdot \nabla v_{\text {ext }}(\mathbf{r})\right\}\left\{\int \gamma^{(2)}\left(\mathbf{r r}^{\prime} ; \mathbf{r r}^{\prime}\right) d^{3} r^{\prime}\right\} d^{3} r}{T\left[\gamma^{(2)}\right]},
\end{aligned}
$$

then $R_{\text {virial }}\left[\tilde{\gamma}_{0}^{(2)}\right]$ takes a value of -2 in the case of $C_{1}=C_{\text {all }}$ :

$$
R_{\text {virial }}\left[\tilde{\gamma}_{0}^{(2)}\right]=-2, \quad \text { if } C_{1}=C_{\text {all }} .
$$

Next we consider the latter case [Fig. 1(b)], namely the case of $C_{1} \subset C_{\text {all }}$. Since $C_{1}$ incompletely covers $C_{\text {all }}$ in the latter case, $C_{2}$ is not always included in $C_{1}$. Therefore, $E\left[\tilde{\gamma}_{0, \lambda}^{(2)}\right]$ does not always take the minimum at $\lambda=1$ as opposed to the former case. Namely, we have

$$
\left.\frac{d E\left[\tilde{\gamma}_{0, \lambda}^{(2)}\right]}{d \lambda}\right|_{\lambda=1} \neq 0
$$

This leads to

$$
R_{\text {virial }}\left[\tilde{\gamma}_{0}^{(2)}\right] \neq-2, \quad \text { if } C_{1} \subset C_{\text {all }} .
$$

This inequality is in stark contrast to Eq. (16). From Eqs. (16) and (18), the theorem concerning the search region of PDs holds:

"If $R_{\text {virial }}\left[\tilde{\gamma}_{0}^{(2)}\right]$ deviates from the exact value of -2 , then the search region of PDs is incomplete."

As the contrapositive, this theorem is restated as follows:

"If the search region of PDs is fully extended, $R_{\text {virial }}\left[\tilde{\gamma}_{0}^{(2)}\right]$ is identical with $-2 . "$

It should be noted that this theorem can be applicable so long as the approximate form of $T\left[\gamma^{(2)}\right]$ satisfies the exact relation Eq. (5). Furthermore, note that the inverse statement of the above theorem is not always satisfied.

This theorem is useful in judging whether the search region of PDs is sufficient or not. We can judge the incompleteness of the search region only by calculating the virial ratio $R_{\text {virial }}\left[\tilde{\gamma}_{0}^{(2)}\right]$. The concrete steps to use this theorem are as follows:

(i) We first calculate the virial ratio $R_{\text {virial }}\left[\tilde{\gamma}_{0}^{(2)}\right]$. (ii) If $R_{\text {virial }}\left[\tilde{\gamma}_{0}^{(2)}\right]$ deviates from -2 , it is found from the theorem that the search region of PDs is insufficient.

(iii) Then, we have to develop and use some scheme which makes the search region of PDs be extended within the set $C_{\text {all }}$. In the next subsection, we shall present the scaling method for the PD functional theory as a promising scheme to extend the search region.

\section{B. Extension of the search region of PDs: Scaling method}

The inequality (17) means that $E\left[\tilde{\gamma}_{0, \lambda}^{(2)}\right]$ may take a minimum value at the PD that belongs to $\bar{C}_{1} \cap C_{2}$, where $\bar{C}_{1}$ denotes the complementary set of $C_{1}$. If we add to the search region the set of PDs that belong to $\bar{C}_{1} \cap C_{2}$, i.e., we retake $C_{1} \cup C_{2}$ as the search region of PDs, then the variationally best PD within $C_{1} \cup C_{2}$ can be obtained from

$$
\left.\frac{d E\left[\tilde{\gamma}_{0, \lambda}^{(2)}\right]}{d \lambda}\right|_{\lambda=\Lambda}=0,
$$

where $\Lambda$ is the optimum value at which $E\left[\tilde{\gamma}_{0, \lambda}^{(2)}\right]$ takes a minimum [Fig. 1(b)]. Namely, the variationally best PD within the substantially extended search region, $C_{1} \cup C_{2}$, is given by $\tilde{\gamma}_{0, \Lambda}^{(2)}$, which is referred to as the "corrected PD." Using Eqs. (5), (8), and (9), Eq. (19) is rewritten by

$$
\begin{aligned}
& 2 \Lambda T\left[\tilde{\gamma}_{0}^{(2)}\right]+W\left[\tilde{\gamma}_{0}^{(2)}\right]-\frac{2}{\Lambda^{2}(N-1)} \int\left\{\mathbf{r} \cdot \nabla_{\mathbf{r} / \Lambda} v_{\mathrm{ext}}(\mathbf{r} / \Lambda)\right\} \\
& \quad \times\left\{\int \tilde{\gamma}_{0}^{(2)}\left(\mathbf{r r}^{\prime} ; \mathbf{r r}^{\prime}\right) d^{3} r^{\prime}\right\} d^{3} r=0 .
\end{aligned}
$$

In the case of the isolated atomic system, by using Eq. (10) instead of Eq. (9), Eq. (19) is easily rewritten as

$$
\Lambda=-\frac{W\left[\tilde{\gamma}_{0}^{(2)}\right]+V\left[\tilde{\gamma}_{0}^{(2)}\right]}{2 T\left[\tilde{\gamma}_{0}^{(2)}\right]} .
$$

Figure 2 shows the flow chart of the scaling method. The scaling method starts with the calculations of the seed PD $\tilde{\gamma}_{0}^{(2)}$ and the corresponding energies, $T\left[\tilde{\gamma}_{0}^{(2)}\right], W\left[\tilde{\gamma}_{0}^{(2)}\right]$, and $V\left[\tilde{\gamma}_{0}^{(2)}\right]$. Using these results, the value of $\Lambda$ is determined from Eq. (20) or (21). Using this $\Lambda$, we get the corrected PD $\tilde{\gamma}_{0, \Lambda}^{(2)}$ from Eq. (3). If the initial search region $C_{1}$ is small compared to $C_{\text {all }}$, the value of $\Lambda$ would generally deviate from 1.0. The corresponding energies, $T\left[\tilde{\gamma}_{0, \Lambda}^{(2)}\right], W\left[\tilde{\gamma}_{0, \Lambda}^{(2)}\right]$, and $V\left[\tilde{\gamma}_{0, \Lambda}^{(2)}\right]$, can also be calculated from Eqs. (5), (8), and (9), respectively. Here note that the load of these calculations is quite light since

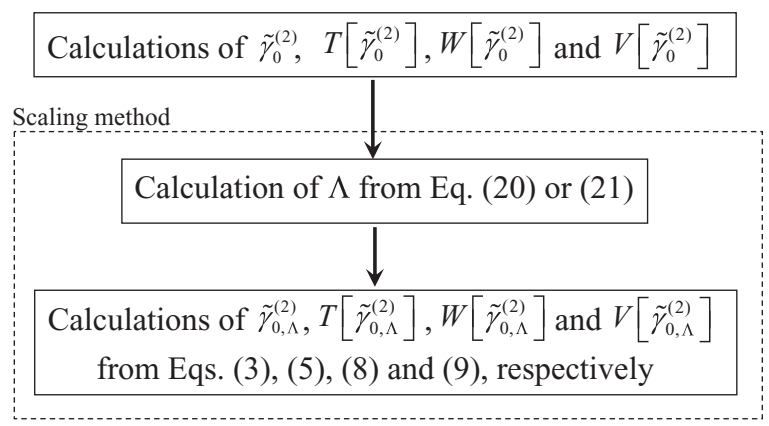

FIG. 2. Flow chart of the scaling method. 
$T\left[\tilde{\gamma}_{0, \Lambda}^{(2)}\right], W\left[\tilde{\gamma}_{0, \Lambda}^{(2)}\right]$, and $V\left[\tilde{\gamma}_{0, \Lambda}^{(2)}\right]$ are easily calculated using already available values of $T\left[\tilde{\gamma}_{0}^{(2)}\right], W\left[\tilde{\gamma}_{0}^{(2)}\right], V\left[\tilde{\gamma}_{0}^{(2)}\right]$, and $\Lambda$.

\section{Formal features of the scaling method}

The above-mentioned scaling method has three formal features. One is that the corrected PD is necessarily $N$ representable if the seed $\mathrm{PD}$ is $N$-representable. This is because the corrected PD is the variationally best PD within the extended search region $\left(C_{1} \cup C_{2}\right)$ that consists of $N$ representable PDs. The second formal feature is that the scaling method does not need to add heavy calculation tasks. As mentioned in Sec. II B, $\tilde{\gamma}_{0, \Lambda}^{(2)}$ and related quantities such as $T\left[\tilde{\gamma}_{0, \Lambda}^{(2)}\right], W\left[\tilde{\gamma}_{0, \Lambda}^{(2)}\right]$, and $V\left[\tilde{\gamma}_{0, \Lambda}^{(2)}\right]$ can be easily calculated only by using the seed PD. The third formal feature is that the corrected PD necessarily satisfies the virial theorem. Namely, we have

$$
R_{\text {virial }}\left[\tilde{\gamma}_{0, \Lambda}^{(2)}\right]=-2
$$

This striking feature can be proven as follows. Multiplying $\Lambda$ on both sides of Eq. (20), using Eqs. (5) and (8), and transforming the integration variable in the third term of the left-hand side, we get

$$
\begin{aligned}
& 2 T\left[\tilde{\gamma}_{0, \Lambda}^{(2)}\right]+W\left[\tilde{\gamma}_{0, \Lambda}^{(2)}\right]-\frac{2}{N-1} \int\left\{\mathbf{r} \cdot \nabla v_{\text {ext }}(\mathbf{r})\right\} \\
& \quad \times\left\{\Lambda^{3} \int \tilde{\gamma}_{0}^{(2)}\left(\Lambda \mathbf{r} \mathbf{r}^{\prime} ; \Lambda \mathbf{r} \mathbf{r}^{\prime}\right) d^{3} r^{\prime}\right\} d^{3} r=0 .
\end{aligned}
$$

On the other hand, integrating both sides of Eq. (3) with respect to $\mathbf{r}^{\prime}$, and transforming the integration variable, we have $\lambda^{3} \int \tilde{\gamma}_{0}^{(2)}\left(\lambda \mathbf{r} \mathbf{r}^{\prime} ; \lambda \mathbf{r} \mathbf{r}^{\prime}\right) d^{3} r^{\prime}=\int \tilde{\gamma}_{0, \lambda}^{(2)}\left(\mathbf{r r}^{\prime} ; \mathbf{r r}^{\prime}\right) d^{3} r^{\prime}$. Substitution of this relation into Eq. (23) results in

$$
\begin{aligned}
& 2 T\left[\tilde{\gamma}_{0, \Lambda}^{(2)}\right]+W\left[\tilde{\gamma}_{0, \Lambda}^{(2)}\right]-\frac{2}{N-1} \int\left\{\mathbf{r} \cdot \nabla v_{\mathrm{ext}}(\mathbf{r})\right\} \\
& \quad \times\left\{\int \tilde{\gamma}_{0, \Lambda}^{(2)}\left(\mathbf{r r}^{\prime} ; \mathbf{r r}^{\prime}\right) d^{3} r^{\prime}\right\} d^{3} r=0 .
\end{aligned}
$$

This equation immediately leads to Eq. (22). Thus, the virial theorem holds rigorously for the corrected PD.

\section{APPROXIMATE FORM OF THE KE FUNCTIONAL}

In this section, we explain how to develop the approximate form of the KE functional that is indispensable for the PD functional theory. In order to bring out the formal features of the scaling method, especially the third feature mentioned in Sec. II C, the approximate form is required to satisfy the exact relation Eq. (5). The strategy we used for the development is (a), which has been described in Sec. I. In this strategy, it seems to be physically desirable that the strong restrictive (necessary) condition and/or a lot of restrictive conditions are satisfied with the approximate KE functional. In the present work, specifically, two kinds of relations and bounds are utilized as restrictive conditions. One is the exact relation Eq. (5), and the other is the exact relation that is derived by means of the Hohenberg-Kohn theorem of the PD functional theory [33].

By applying $\lim _{\lambda \rightarrow 1} \frac{\partial}{\partial \lambda}$ to both sides of Eq. (5), and using the fact that integrals over an infinitely distant surface vanish in the isolated system, we have [33]

$$
\begin{aligned}
2 T\left[\gamma^{(2)}\right]= & -\iint \mathbf{r} \cdot\left\{\nabla \frac{\delta T\left[\gamma^{(2)}\right]}{\delta \gamma^{(2)}\left(\mathbf{r} \mathbf{r}^{\prime} ; \mathbf{r r} \mathbf{r}^{\prime}\right)}\right\} \gamma^{(2)}\left(\mathbf{r} \mathbf{r}^{\prime} ; \mathbf{r r}^{\prime}\right) d^{3} r d^{3} r^{\prime} \\
& -\iint \mathbf{r}^{\prime} \cdot\left\{\nabla^{\prime} \frac{\delta T\left[\gamma^{(2)}\right]}{\delta \gamma^{(2)}\left(\mathbf{r} \mathbf{r}^{\prime} ; \mathbf{r} \mathbf{r}^{\prime}\right)}\right\} \gamma^{(2)}\left(\mathbf{r} \mathbf{r}^{\prime} ; \mathbf{r} \mathbf{r}^{\prime}\right) d^{3} r d^{3} r^{\prime},
\end{aligned}
$$

where $\nabla$ and $\nabla^{\prime}$ denote the nabla operators with respect to $\mathbf{r}$ and $\mathbf{r}^{\prime}$, respectively. This is the form of the exact relation used in developing the approximate form.

Using the Hohenberg-Kohn theorem of the PD functional theory [33], the other relation for the KE functional can be obtained as

$$
\left.\iint \gamma_{0}^{(2)}\left(\mathbf{r r}^{\prime} ; \mathbf{r r}^{\prime}\right) \frac{\delta T\left[\gamma^{(2)}\right]}{\delta \gamma^{(2)}\left(\mathbf{r r}^{\prime} ; \mathbf{r r}^{\prime}\right)}\right|_{\gamma^{(2)}=\gamma_{0}^{(2)}} d^{3} r d^{3} r^{\prime}=T\left[\gamma_{0}^{(2)}\right]
$$

where the functional derivative in the left-hand side should be calculated by varying the PD with keeping the $N$ representability [33].

As shown in the previous papers [33,34], Eq. (26) is fulfilled if the $\mathrm{KE}$ functional is given by the following form:

$$
T\left[\gamma^{(2)}\right]=\iint f\left(\mathbf{r}, \mathbf{r}^{\prime}\right) \gamma^{(2)}\left(\mathbf{r} \mathbf{r}^{\prime} ; \mathbf{r r}^{\prime}\right) d^{3} r d^{3} r^{\prime},
$$

where $f\left(\mathbf{r}, \mathbf{r}^{\prime}\right)$ is an arbitrary function, and should be determined by the other condition. Substitution of Eq. (27) into Eq. (25) leads to the condition for $f\left(\mathbf{r}, \mathbf{r}^{\prime}\right)$ :

$$
\begin{aligned}
& \iint\left\{2 f\left(\mathbf{r}, \mathbf{r}^{\prime}\right)+\mathbf{r} \cdot \nabla f\left(\mathbf{r}, \mathbf{r}^{\prime}\right)+\mathbf{r}^{\prime} \cdot \nabla^{\prime} f\left(\mathbf{r}, \mathbf{r}^{\prime}\right)\right\} \\
& \times \gamma^{(2)}\left(\mathbf{r} \mathbf{r}^{\prime} ; \mathbf{r r}^{\prime}\right) d^{3} r d^{3} r^{\prime}=0 .
\end{aligned}
$$

Note that if $f\left(\mathbf{r}, \mathbf{r}^{\prime}\right)$ is determined by requiring it to satisfy Eq. (28), then the KE functional, i.e., Eq. (27) with such $f\left(\mathbf{r}, \mathbf{r}^{\prime}\right)$, fulfills both Eqs. (25) and (26).

In order to determine $f\left(\mathbf{r}, \mathbf{r}^{\prime}\right)$ by means of Eq. (28), we utilize the following equation that is satisfied by an arbitrary $N$-representable PD;

$$
\iint G\left(\mathbf{r}, \mathbf{r}^{\prime}\right) \gamma^{(2)}\left(\mathbf{r} \mathbf{r}^{\prime} ; \mathbf{r r}^{\prime}\right) d^{3} r d^{3} r^{\prime}=0,
$$

where $G\left(\mathbf{r}, \mathbf{r}^{\prime}\right)$ is an arbitrary antisymmetric function. From Eqs. (28) and (29), we have

$$
2 f\left(\mathbf{r}, \mathbf{r}^{\prime}\right)+\mathbf{r} \cdot \nabla f\left(\mathbf{r}, \mathbf{r}^{\prime}\right)+\mathbf{r}^{\prime} \cdot \nabla^{\prime} f\left(\mathbf{r}, \mathbf{r}^{\prime}\right)=G\left(\mathbf{r}, \mathbf{r}^{\prime}\right) .
$$

It is preferable that $G\left(\mathbf{r}, \mathbf{r}^{\prime}\right)$ is chosen on the basis of some physically sound reason. For this aim, we here adopt the condition that the total force acting on the system is zero, i.e., [58]

$$
\int \rho(\mathbf{r}) \nabla v_{\mathrm{ext}}(\mathbf{r}) d^{3} r=0,
$$

where $\rho(\mathbf{r})$ denotes the electron density that is given by $\rho(\mathbf{r})=$ $\frac{2}{N-1} \int \gamma^{(2)}\left(\mathbf{r r}^{\prime} ; \mathbf{r r}^{\prime}\right) d^{3} r^{\prime}$. By using the symmetric property of PDs, i.e., $\gamma^{(2)}\left(\mathbf{r} \mathbf{r}^{\prime} ; \mathbf{r r}^{\prime}\right)=\gamma^{(2)}\left(\mathbf{r}^{\prime} \mathbf{r} ; \mathbf{r}^{\prime} \mathbf{r}\right)$, Eq. (31) is rewritten as $\iint\left\{\nabla v_{\text {ext }}(\mathbf{r})-\nabla^{\prime} v_{\text {ext }}\left(\mathbf{r}^{\prime}\right)\right\} \gamma^{(2)}\left(\mathbf{r} \mathbf{r}^{\prime} ; \mathbf{r r}^{\prime}\right) d^{3} r d^{3} r^{\prime}=0$. Choosing the external potential of the isolated atomic system as $v_{\text {ext }}(\mathbf{r})$, 
and considering the force in the $z$ direction, we get

$$
\iint\left\{\frac{\cos \theta}{r^{2}}-\frac{\cos \theta^{\prime}}{r^{\prime 2}}\right\} \gamma^{(2)}\left(\mathbf{r} \mathbf{r}^{\prime} ; \mathbf{r r}^{\prime}\right) d^{3} r d^{3} r^{\prime}=0,
$$

where $\theta$ and $\theta^{\prime}$ stand for polar angles of $\mathbf{r}$ and $\mathbf{r}^{\prime}$, respectively. Comparing Eq. (29) with Eq. (32), we may choose the following function as $G\left(\mathbf{r}, \mathbf{r}^{\prime}\right)$ :

$$
G\left(\mathbf{r}, \mathbf{r}^{\prime}\right)=\frac{\cos \theta}{r^{2}}-\frac{\cos \theta^{\prime}}{r^{\prime 2}} .
$$

Substituting Eq. (33) into Eq. (30), and solving Eq. (30), we have

$$
\begin{aligned}
f\left(\mathbf{r}, \mathbf{r}^{\prime}\right)= & K\left(\frac{1}{2 r^{2}}+\frac{1}{2 r^{\prime 2}}\right)+\left(\frac{\cos \theta}{r^{2}}-\frac{\cos \theta^{\prime}}{r^{\prime 2}}\right) \ln r^{\prime} \\
& +a \frac{\cos \theta}{r^{2}} \ln \left(\frac{r}{r^{\prime}}\right)+b \frac{\cos \theta^{\prime}}{r^{\prime 2}} \ln \left(\frac{r^{\prime}}{r}\right),
\end{aligned}
$$

where $K, a$, and $b$ are arbitrary constants. This solution is a particular solution of the partial differential equation (30). It should be noted that the general solution of Eq. (30) can be obtained by the method of characteristics [59]. The general solution is given by

$$
f\left(\mathbf{r}, \mathbf{r}^{\prime}\right)=\left(\frac{\cos \theta}{r^{2}}-\frac{\cos \theta^{\prime}}{r^{\prime 2}}\right) \ln r^{\prime}+\frac{1}{r^{\prime 2}} g\left(\frac{r^{\prime}}{r}\right),
$$

where $g\left(r^{\prime} / r\right)$ is an arbitrary function of $r^{\prime} / r$. It is easily confirmed that Eq. (34) corresponds to a particular form of Eq. (35).

It is obvious that Eq. (34) thus determined satisfies Eq. (28) and provides a promising form of $T\left[\gamma^{(2)}\right]$ which satisfies Eqs. (25) and (26). However, it is also noticed that we can choose as $f\left(\mathbf{r}, \mathbf{r}^{\prime}\right)$ the other types of functions which satisfy Eq. (28). Specifically, as can be seen from the above derivation, it is possible to choose $G\left(\mathbf{r}, \mathbf{r}^{\prime}\right)$ and $g\left(r^{\prime} / r\right)$ which are different from the present functions, i.e., Eq. (33) and $g\left(r^{\prime} / r\right)$ in Eq. (34), respectively. In order to judge whether the choices of these functions are appropriate or not, there is nothing for it but to perform the numerical calculations or check them by the other restrictive conditions which differ from two ones used here. As shown later by numerical calculations, the present choices of $G\left(\mathbf{r}, \mathbf{r}^{\prime}\right)$ and $g\left(r^{\prime} / r\right)$ lead to reasonable results. The details are presented in Sec. IV.

Substituting Eq. (34) into Eq. (27), and using the symmetric property $\gamma^{(2)}\left(\mathbf{r r}^{\prime} ; \mathbf{r r}^{\prime}\right)=\gamma^{(2)}\left(\mathbf{r}^{\prime} \mathbf{r} ; \mathbf{r}^{\prime} \mathbf{r}\right)$, we get the approximate form [34],

$$
\begin{aligned}
T\left[\gamma^{(2)}\right]= & \iint\left[K\left(\frac{1}{r^{2}}+\frac{1}{r^{\prime 2}}\right)\right. \\
& \left.+K^{\prime}\left\{\frac{\cos \theta}{2 r^{2}} \ln \left(\frac{r}{r^{\prime}}\right)+\frac{\cos \theta^{\prime}}{2 r^{\prime 2}} \ln \left(\frac{r^{\prime}}{r}\right)\right\}\right] \\
& \times \gamma^{(2)}\left(\mathbf{r} \mathbf{r}^{\prime} ; \mathbf{r r}^{\prime}\right) d^{3} r d^{3} r^{\prime}
\end{aligned}
$$

where $K^{\prime}$ is the arbitrary constant that is related to $a$ and $b$ by $K^{\prime}=a+b-1$. In the next section, we explain how to determine the values of $K$ and $K^{\prime}$ in conjunction with details of the calculation method.
TABLE I. Total numbers of SDs (NSD) used in atomic structure calculations.

\begin{tabular}{lrrr}
\hline \hline & $\mathrm{Ne}$ & $\mathrm{Mg}$ & \multicolumn{1}{c}{$\mathrm{Ar}$} \\
\hline NSD in constructing PDs of the set $C_{1}$ & 1821 & 2569 & 3167 \\
NSD in the scaling method & 1821 & 2569 & 3167 \\
Maximum NSD & 10478 & 10285 & 14618 \\
\hline
\end{tabular}

\section{ATOMIC STRUCTURE CALCULATIONS}

\section{A. Details of calculations}

In order to evaluate the scaling method that is combined with Eq. (36), we perform electronic structure calculations for the neutral $\mathrm{Ne}, \mathrm{Mg}$, and Ar atoms. First, we have to determine the seed PD $\tilde{\gamma}_{0}^{(2)}$ and the corresponding energy functionals, $T\left[\tilde{\gamma}_{0}^{(2)}\right], W\left[\tilde{\gamma}_{0}^{(2)}\right]$, and $V\left[\tilde{\gamma}_{0}^{(2)}\right]$. In this paper, to calculate these quantities, we utilize the previously proposed scheme [33]. The search region of the previously proposed scheme [33], which corresponds to the set of $C_{1}$ of the present scheme, consists of PDs that are constructed from the linear combination of SDs. The SDs are prepared by using eigenfunctions of the effective initial scheme [28,30,32] as the constituent singleparticle orbitals. We choose as the basis SDs employed in the linear combination the zero- and doubly excited SDs that have numerically non-negligible contributions to the resultant PD. As a result, the total numbers of SDs (NSD) used in constructing PDs of the set $C_{1}$ are 1821, 2569, and 3167 for the cases of the $\mathrm{Ne}, \mathrm{Mg}$, and $\mathrm{Ar}$ atoms, respectively. The calculation conditions are summarized in Table I.

After getting $\tilde{\gamma}_{0}^{(2)}, T\left[\tilde{\gamma}_{0}^{(2)}\right], W\left[\tilde{\gamma}_{0}^{(2)}\right]$, and $V\left[\tilde{\gamma}_{0}^{(2)}\right]$, we calculate the corrected PD $\tilde{\gamma}_{0, \Lambda}^{(2)}$ and the corresponding energy functionals, $T\left[\tilde{\gamma}_{0, \Lambda}^{(2)}\right], W\left[\tilde{\gamma}_{0, \Lambda}^{(2)}\right]$, and $V\left[\tilde{\gamma}_{0, \Lambda}^{(2)}\right]$, along the way illustrated in Fig. 2. We also evaluate the virial ratios, electron densities, and xc holes in order to check the validity of the scaling method. To highlight the efficiency of the scaling method in extending the search region, we further perform numerical calculations by using the other search regions that are extended by naively increasing the NSD without the scaling method. The maximum NSDs adopted here are 10 478, 10285 , and 14618 for the cases of the $\mathrm{Ne}, \mathrm{Mg}$, and $\mathrm{Ar}$ atoms, respectively (Table I).

Let us explain how to determine the values of $K$ and $K^{\prime}$ that appear in Eq. (36). These are determined by two conditions. One is that Eq. (36) should be coincident with the Hartree-Fock KE when the Hartree-Fock PD is substituted in it. The second condition is that among the pairs $\left(K, K^{\prime}\right)$ that satisfy the first condition, we will choose the pair $\left(K, K^{\prime}\right)$ that minimizes the root-mean-square error (RMSE) of the external potential and electron-electron interaction energies. Thus, the values of $K$ and $K^{\prime}$ are determined by requiring the approximate functional to have these two sound features.

\section{B. Calculation results}

\section{Errors of the external potential and electron-electron interaction energies}

Since both the external potential and electron-electron interaction energies are exactly calculated from the PD, the 


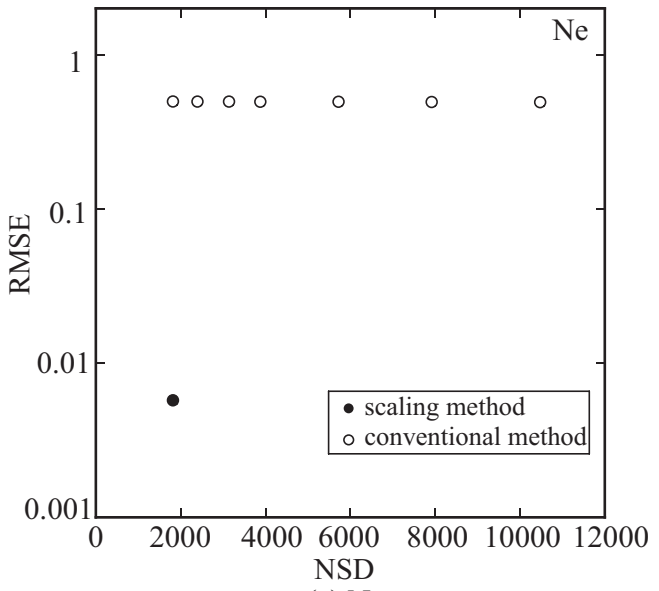

(a) $\mathrm{Ne}$

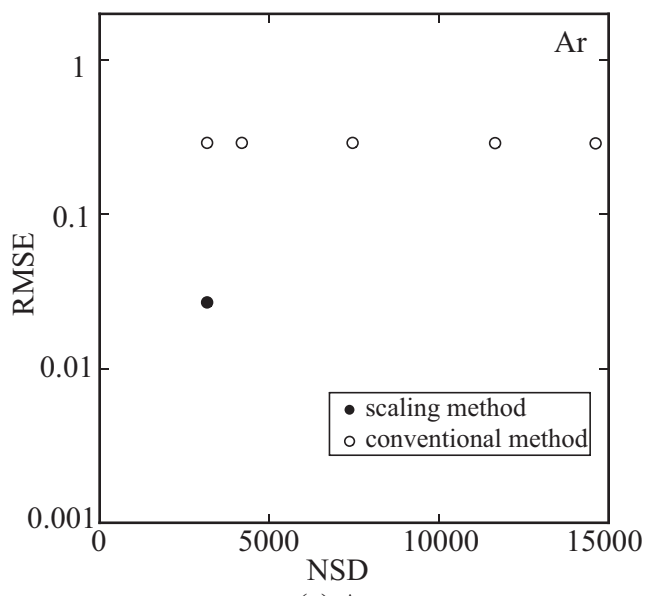

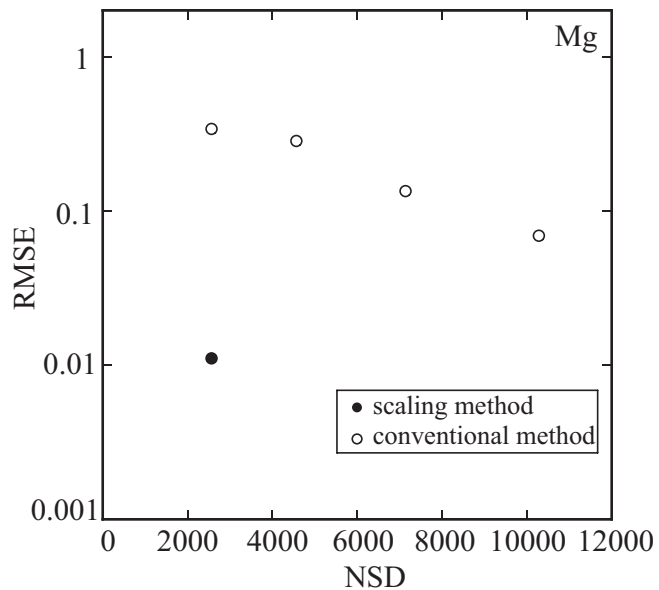

(b) $\mathrm{Mg}$

(c) $\mathrm{Ar}$

FIG. 3. The RMSEs of the external potential and electron-electron interaction energies for the cases of (a) $\mathrm{Ne}$, (b) Mg, and (c) Ar. The solid and open circles denote RMSEs with the scaling method and those with the conventional method, respectively. Details of each method are explained in the text.

errors of the external potential energy and electron-electron interaction energy, i.e., $\Delta V$ and $\Delta W$, indicate the error of the PD directly. Therefore, the RMSE of the external potential and electron-electron interaction energies, which is defined by $\sqrt{\left\{(\Delta V)^{2}+(\Delta W)^{2}\right\} / 2}$, can be regarded as a good benchmark of the accuracy of the PD. The error is estimated by $\Delta A=$ ( $\left.A_{\text {present }}-A_{\text {reference }}\right) / A_{\text {reference }}$, where $A$ corresponds to the external potential energy, electron-electron interaction energy or etc., and where $A_{\text {present }}$ and $A_{\text {reference }}$ denote the present result and the reference data, respectively. In calculating $\Delta V$ and $\Delta W$, we adopt as the reference data the results of the $1 / Z$ expansion method $[60,61]$ that is based on the data by Davidson and coworkers [62,63]. The results are shown as a function of the NSD in Figs. 3(a)-3(c). In these figures, results done by the scaling method, i.e., RMSE of $W\left[\tilde{\gamma}_{0, \Lambda}^{(2)}\right]$ and $V\left[\tilde{\gamma}_{0, \Lambda}^{(2)}\right]$, are denoted as "scaling method" (solid circles), and results done by the method which is accompanied with naively increasing NSD and without the scaling method, i.e., RMSE of $W\left[\tilde{\gamma}_{0}^{(2)}\right]$ and $V\left[\tilde{\gamma}_{0}^{(2)}\right]$, are denoted as "conventional method" (open circles). It is found that the scaling method much reduces RMSEs, the reduction rate of which are about $1 / 100,1 / 30$, and $1 / 10$ for the $\mathrm{Ne}, \mathrm{Mg}$, and Ar atoms, respectively. Although
RMSEs are improved also by the conventional method, the reduction rates are worse than those by the scaling method. For instance, RMSEs for $\mathrm{Ne}$ and $\mathrm{Ar}$ are down only about $1 \%$ even though the NSD increases over 10000 [Figs. 3(a) and $3(\mathrm{c})]$.

In order to inquire the RMSE further in more details, we show $\Delta W$ and $\Delta V$ individually for the $\mathrm{Ne}, \mathrm{Mg}$, and $\mathrm{Ar}$ atoms in Figs. 4, 5, and 6, respectively. For each atom, both $\Delta W$ and $\Delta V$ are more reduced by the scaling method than by the conventional method. We can say from these figures that the improvements in RMSEs are achieved through the reductions in both $\Delta W$ and $\Delta V$. This implies that the seed PD itself is improved reasonably by the scaling method.

Here, we shall comment on dependencies of the RMSE, $\Delta W$ and $\Delta V$ on the NSD for $\mathrm{Mg}$, which are a bit different from those for $\mathrm{Ne}$ and $\mathrm{Ar}$ (Figs. 3-6). In general, to what extent the search region of PDs is extended by the conventional method depends on the choice of the basis SDs. It is found from Figs. 3-6 that the search region of PDs for the case of $\mathrm{Mg}$ is extended relatively well, as compared with the cases of $\mathrm{Ne}$ and Ar. Nevertheless, it should be noticed from Figs. 3-6 that such the extension by the conventional method is definitely inferior to that by the scaling method. 


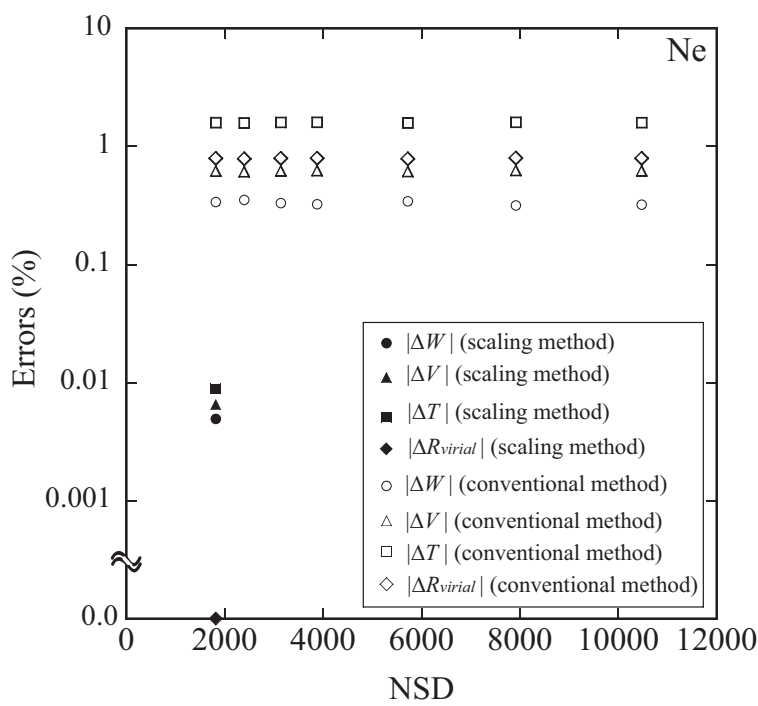

FIG. 4. Errors of the electron-electron interaction energy $(\Delta W)$, external potential energy $(\Delta V), \operatorname{KE}(\Delta T)$, and virial ratio $\left(\Delta R_{\text {virial }}\right)$ for the Ne atom as a function of the NSD. The solid and open symbols denote errors with the scaling method and those with the conventional method, respectively.

Thus, the superior efficiency of the scaling method in extending the search region of PDs is explicitly shown.

\section{Error of the kinetic energy}

The dependencies of the errors of the $\operatorname{KE}(\Delta T)$ on the NSD are also shown in Figs. 4-6. It is found that $\Delta T$ is reduced more effectively by the scaling method than by the conventional method. In general, the accuracy of the KE depends on both the appropriateness of the approximate form of the functional and that of the resultant PD. As mentioned in Sec. IV B-1,

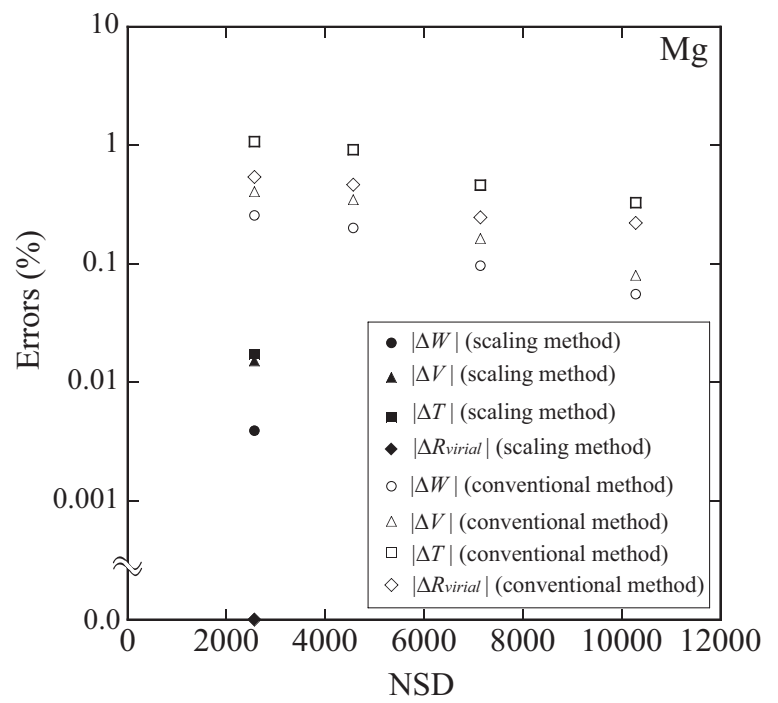

FIG. 5. Errors of the electron-electron interaction energy $(\Delta W)$, external potential energy $(\Delta V), \operatorname{KE}(\Delta T)$, and virial ratio $\left(\Delta R_{\text {virial }}\right)$ for the $\mathrm{Mg}$ atom as a function of the NSD. The solid and open symbols denote errors with the scaling method and those with the conventional method, respectively.

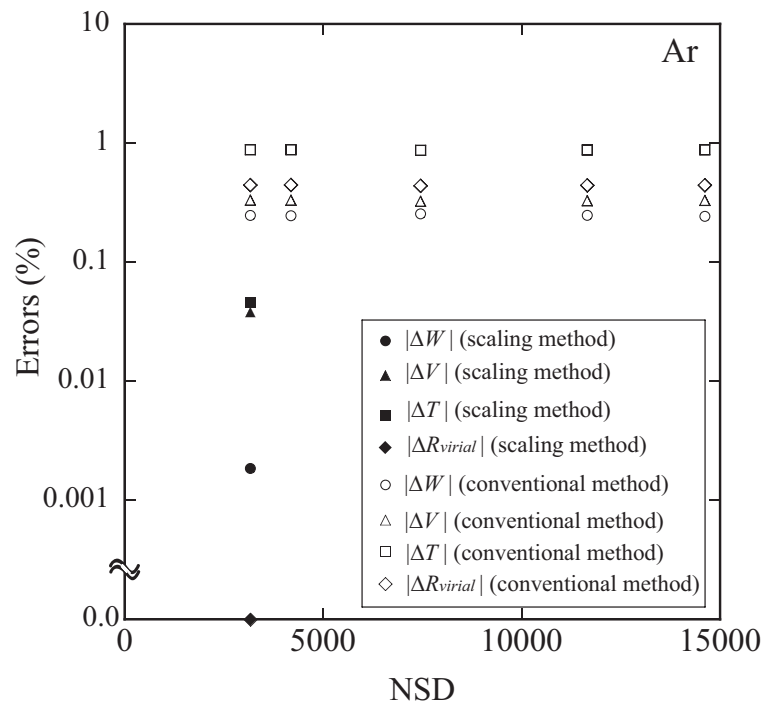

FIG. 6. Errors of the electron-electron interaction energy $(\Delta W)$, external potential energy $(\Delta V), \operatorname{KE}(\Delta T)$, and virial ratio $\left(\Delta R_{\text {virial }}\right)$ for the Ar atom as a function of the NSD. The solid and open symbols denote errors with the scaling method and those with the conventional method, respectively.

$\tilde{\gamma}_{0, \Lambda}^{(2)}$ seems to be reproduced appropriately. Therefore, the improvement of the KE suggests that the approximate form itself, which is given by Eq. (36), would be also sound.

In this work, the coefficients $K$ and $K^{\prime}$ for Eq. (36) are determined along the way mentioned in Sec. IV A. If Eq. (36) includes the form close to the correct $\mathrm{KE}$ functional and if the search region of PDs is sufficiently extended, thus determined $K$ and $K^{\prime}$ are expected to become the universal constants on their own. The resultant $K$ and $K^{\prime}$ of this work take the values ranging $0.0423-0.0688(K)$, and $0.125-0.360\left(K^{\prime}\right)$, respectively. Comparing to the approximate forms of the $\mathrm{xc}$ energy functional of the conventional DFT [64], the present functional is not so far from the universal one, and it has a promising form though there is still room for improvement.

\section{Virial ratio}

As mentioned in Sec. II A, the deviation of the virial ratio from -2 implies the incompleteness of the search region of PDs. The errors of the virial ratio $\left(\Delta R_{\text {virial }}\right)$ for the $\mathrm{Ne}, \mathrm{Mg}$, and $\mathrm{Ar}$ atoms are shown in Figs. 4, 5, and 6, respectively. It is found from Figs. $4-6$ that $R_{\text {virial }}\left[\tilde{\gamma}_{0}^{(2)}\right]$ (the virial ratio by the conventional method) deviates from the correct value of -2 even though the search region of PDs is extended by increasing the NSD. This means that the search region is not always extended efficiently by increasing the NSD naively. A huge number of SDs would be needed for satisfying the virial theorem if the conventional method was used in extending the search region of PDs. On the other hand, $R_{\text {virial }}\left[\tilde{\gamma}_{0, \Lambda}^{(2)}\right]$ (the virial ratio by the scaling method) is rigorously equal to -2 , as mentioned in Sec. II C. The seed PD can be corrected to the PD that satisfies the virial theorem, along a simple calculation procedure without additional and heavy calculation tasks, as shown in Fig. 2. Thus, also from the viewpoint of the virial 
theorem, the scaling method makes the search region of PDs be extended efficiently.

There seems to be a mutual relation between the virial theorem and improvements of various energy functionals mentioned in the preceding subsections. Namely, the electronelectron interaction energy, external potential energy, and $\mathrm{KE}$ are improved in a well-balanced manner due to the feature of satisfying the virial theorem rigorously.

\section{Electron density}

In the previous subsections, the appropriateness of the corrected PD is shown from the viewpoints of energy functionals and virial ratio. In this and subsequent subsections, to clarify the details of how the PD is spatially improved by the scaling method, we examine the spatial distributions of the electron density (this subsection) and the xc hole (the next subsection).

The electron densities of the $\mathrm{Ne}$ atom that are calculated from $\tilde{\gamma}_{0, \Lambda}^{(2)}$ [scaling method $\left.(\mathrm{NSD}=1821)\right]$ and $\tilde{\gamma}_{0}^{(2)}$ [conventional method $(\mathrm{NSD}=1821$ and 10 478)] are shown in Fig. 7(a), together with the electron density of the configuration interaction (CI) method [67]. The first peak of the electron density with the scaling method is in a better agreement with that of the CI method [67] than those of the electron densities with the conventional method (NSD $=1821$ and 10478 ) [Fig. 7(b)]. On the other hand, the second peak is modified excessively by the scaling method [Fig. 7(c)]. Let us discuss this tendency below.

The scaling method has the property that if $\Lambda$ is larger than 1 as is the present case, then both the first and second peaks

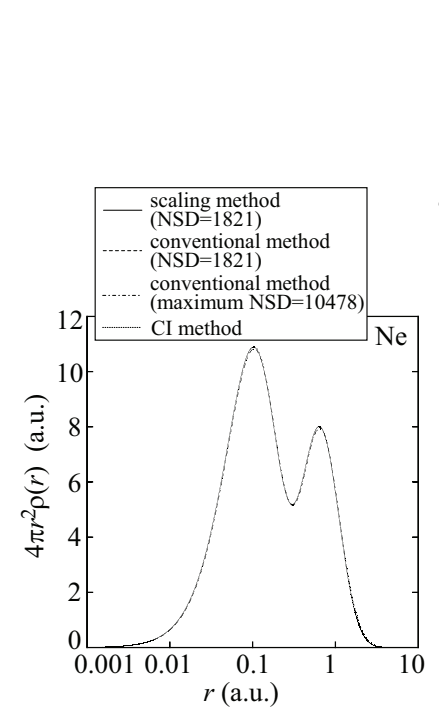

(a)

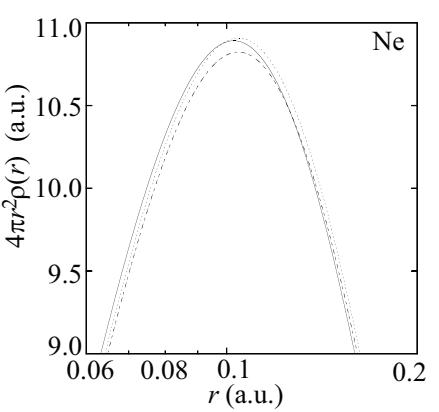

(b)

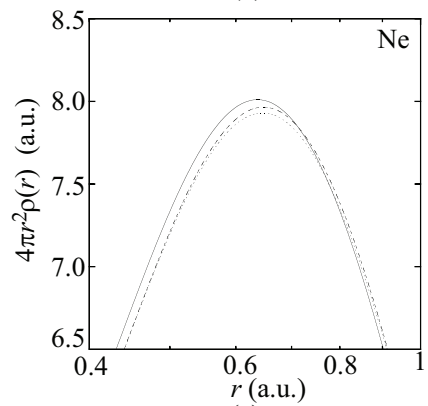

(c)
FIG. 7. Profiles of electron densities of the Ne atom. The solid line denotes electron density calculated with the scaling method $\left[\tilde{\rho}_{0, \Lambda}(\mathbf{r})\right]$. The dashed and chained lines stand for electron densities that are calculated with the conventional method $\left[\tilde{\rho}_{0}(\mathbf{r})\right]$ in the cases of NSD $=1821$ and NSD $=10478$ (maximum), respectively. The density profiles are zoomed in around the first peak (b) and the second peak (c). are simultaneously enhanced by the scaling method. This is because the corrected electron density $\tilde{\rho}_{0, \Lambda}(\mathbf{r})$ is given by

$$
\begin{aligned}
\tilde{\rho}_{0, \Lambda}(\mathbf{r}) & =\frac{2}{N-1} \int \tilde{\gamma}_{0, \Lambda}^{(2)}\left(\mathbf{r r}^{\prime} ; \mathbf{r r}^{\prime}\right) d^{3} r^{\prime} \\
& =\frac{2 \Lambda^{3}}{N-1} \int \tilde{\gamma}_{0}^{(2)}\left(\mathbf{r r}^{\prime} ; \mathbf{r r}^{\prime}\right) d^{3} r^{\prime} \\
& =\Lambda^{3} \tilde{\rho}_{0}(\Lambda \mathbf{r}),
\end{aligned}
$$

where $\tilde{\rho}_{0}(\mathbf{r})$ is the electron density that is calculated from the seed PD $\tilde{\gamma}_{0}^{(2)}$. The enhancement of the first peak [Fig. 7(b)] causes the decrease of $|\Delta V|$, while the excessive modification of the second peak [Fig. 7(c)] causes the increase of $|\Delta V|$. Since it is located closer to the nucleus than the second peak, and since its magnitude is larger than that of the second peak, the first peak contributes to the external potential energy more significantly than the second one. Therefore, it is expected that $|\Delta V|$ decreases due to the improvement of the first peak, and indeed it is much improved as shown in Fig. 4.

The above-mentioned correction of the electron density also has an important role in the improvement of the Hartree energy that is the main term of the electron-electron interaction energy. This is because since the first peak is higher than that of the second peak [Fig. 7(a)], the electron density around the first peak contributes to the Hartree energy more significantly than that around the second peak.

Thus, the scaling method improves the spatial distribution of the electron density in such a way that the errors of the external potential energy and Hartree energy are reduced effectively. Namely, the dominant part (the first peak of the electron density) that largely contributes to both the external potential energy and Hartree energy is preferentially improved by the scaling method.

In the next subsection, we shall mention that the improvement of the Hartree energy leads to the decrease of $|\Delta W|$ in conjunction with discussing the improvement of the xc energy term.

\section{Exchange-correlation hole}

In order to check where the PD is corrected spatially via the scaling method, in this subsection we shall investigate the xc hole $n_{\mathrm{xc}}\left(\mathbf{r}, \mathbf{r}^{\prime}\right)$. The xc hole reflects the spatial shape of the PD explicitly. The xc hole is defined as

$$
n_{\mathrm{xc}}\left(\mathbf{r}, \mathbf{r}^{\prime}\right)=\frac{2 \gamma^{(2)}\left(\mathbf{r} \mathbf{r}^{\prime} ; \mathbf{r} \mathbf{r}^{\prime}\right)-\rho(\mathbf{r}) \rho\left(\mathbf{r}^{\prime}\right)}{\rho(\mathbf{r})} .
$$

The calculation results are shown in Fig. 8 for the $\mathrm{Ne}$ atom and shown in Fig. 9 for the $\mathrm{Mg}$ and Ar atoms. They are calculated from $\tilde{\gamma}_{0, \Lambda}^{(2)}$ (scaling method) and $\tilde{\gamma}_{0}^{(2)}$ (conventional method) under the condition that the reference electron is placed at $z=0.2$ or $z=0.4$. For comparison, the corresponding profiles calculated by the CI method [68] are also shown in Figs. 8(a) and $8(\mathrm{~b})$.

Let us consider the properties of $n_{\mathrm{xc}}\left(\mathbf{r}, \mathbf{r}^{\prime}\right)$ in the case of $z=0.2$, which are shown in Fig. 8(a). Judging from the spatial distribution of each constituent electron of the $\mathrm{Ne}$ atom, the large hole around $z^{\prime}-z=-0.2$ would be firstly due to the Pauli principle between the $1 s$ electrons, and secondly due to the Pauli principles between the $2 s$ electrons, and between 


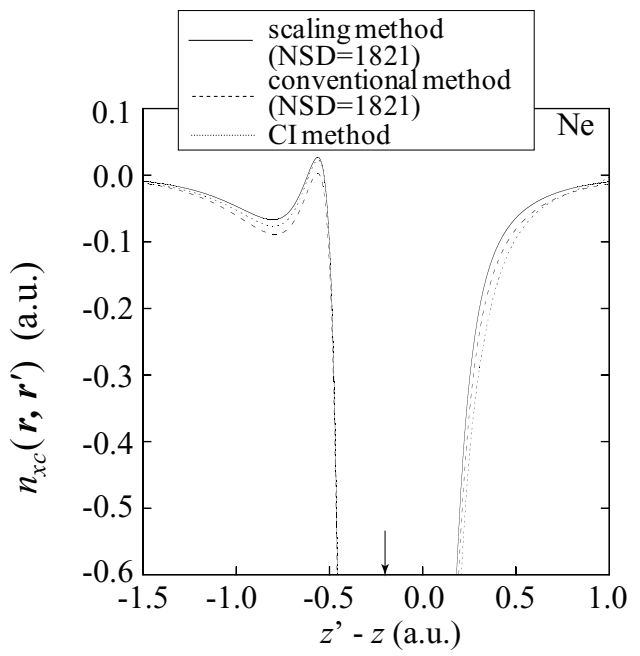

(a) $z=0.2$ (a.u.)

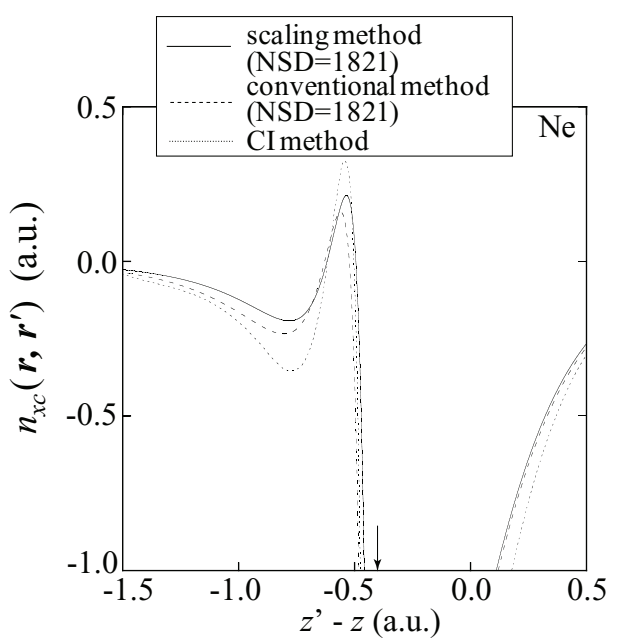

(b) $z=0.4$ (a.u.)

FIG. 8. Profiles of the xc holes $\left[n_{\mathrm{xc}}\left(\mathbf{r}, \mathbf{r}^{\prime}\right)\right]$ of the $\mathrm{Ne}$ atom along $z$ axis. The reference electron is placed at (a) $z=0.2$ or (b) $z=0.4$. The solid and dashed lines denote xc holes calculated with the scaling method and conventional one, respectively. The position of the nucleus is denoted by the arrow.

the $2 p$ electrons. Also, the small hole around $z^{\prime}-z=-0.7$ would be mainly due to the Pauli principle between the $2 s$ electrons. As an example among them, we shall explain the Pauli principle between the $1 s$ electrons. The existence probability of electrons at $z=0.2$ is largely attributed to the $1 s$ electron. Therefore, we have to consider the Pauli principle between the $1 s$ electrons at $z=0.2$. Since the $1 s$ electron is large near the nucleus, i.e., $z^{\prime}=0$, the xc hole becomes large near $z^{\prime}-z=-0.2$.

Similarly to the above case, properties of $n_{\mathrm{xc}}\left(\mathbf{r}, \mathbf{r}^{\prime}\right)$ in the case of $z=0.4$ [Fig. 8(b)] can be described as follows. The large hole observed around $z^{\prime}-z=-0.2$ would be firstly due to the Pauli principle between the $2 p$ electrons, and secondly due to the Pauli principles between the $1 s$ electrons and between the $2 s$ electrons. The small hole around $z^{\prime}-z=-0.8$ would be due to the Pauli principles between the $2 p$ electrons and between the $2 s$ electrons, to the same degree.

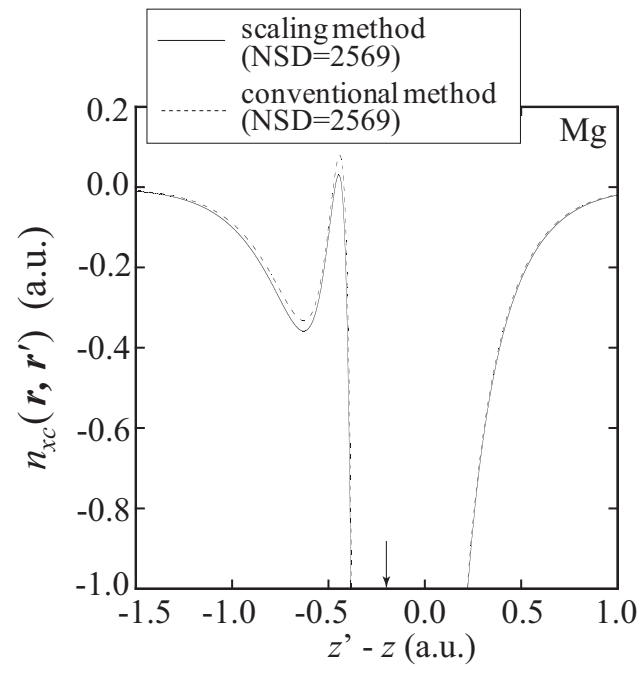

(a) $z=0.2$ (a.u.)

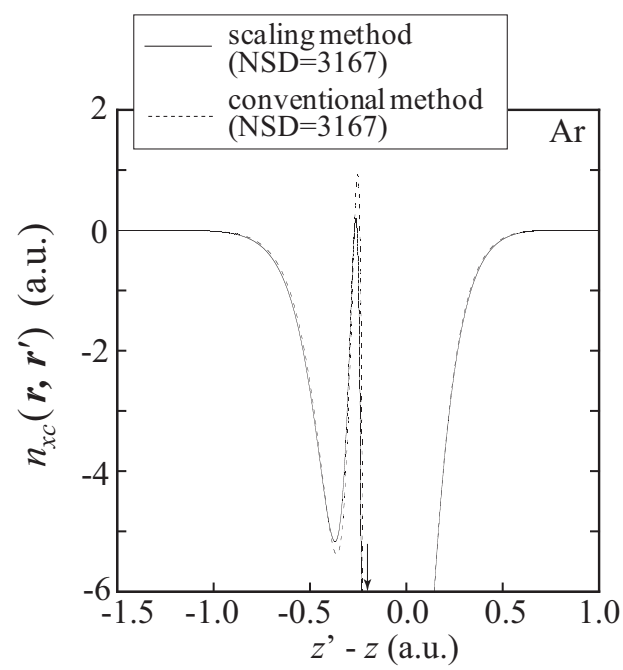

(b) $z=0.2$ (a.u.)

FIG. 9. Profiles of the xc holes $\left[n_{\mathrm{xc}}\left(\mathbf{r}, \mathbf{r}^{\prime}\right)\right]$ along $z$ axis for (a) $\mathrm{Mg}$ and (b) Ar, respectively. The reference electron is placed at $z=0.2$. The solid and dashed lines denote $\mathrm{xc}$ holes calculated with the scaling method and conventional one, respectively. The position of the nucleus is denoted by the arrow.

It is found from Fig. 8(a) $(z=0.2)$ that the xc hole profile calculated with the scaling method is in a better agreement with the corresponding CI result than that calculated with the conventional method. On the other hand, it is found from Fig. 8(b) $(z=0.4)$ that the peak height around $z^{\prime}-z=-0.6$ is improved by the scaling method while the shape of the valley around $z^{\prime}-z=-0.8$ is not improved.

It is shown that the accuracy of the xc hole at $z=0.2$ affects the reproducibility of the xc energy more strongly than that at $z=0.4$. To confirm this, we consider the usual relation between the xc hole and xc energy $\varepsilon_{\mathrm{xc}}$;

$$
\varepsilon_{\mathrm{xc}}=\frac{e^{2}}{2} \iint \frac{\rho(\mathbf{r}) n_{\mathrm{xc}}\left(\mathbf{r}, \mathbf{r}^{\prime}\right)}{\left|\mathbf{r}-\mathbf{r}^{\prime}\right|} d^{3} r d^{3} r^{\prime} .
$$

From this equation, the integration region, where $\rho(\mathbf{r})$ and $1 /\left|\mathbf{r}-\mathbf{r}^{\prime}\right|$ that appear in the integrand are large, makes a 
large contribution to the $\mathrm{xc}$ energy. Since $\rho(\mathbf{r})$ is large near the nucleus $(\mathbf{r}=0)$, the region that satisfies the above condition is the vicinity of $\mathbf{r}=\mathbf{r}^{\prime}=0$. This means that the accuracy of $n_{\mathrm{xc}}\left(\mathbf{r}, \mathbf{r}^{\prime}\right)$ at $z=0.2$ is more important than that at $z=0.4$ in evaluating the xc energy. Thus, the scaling method would preferentially improve the spatial parts of $n_{\mathrm{xc}}\left(\mathbf{r}, \mathbf{r}^{\prime}\right)$, the accuracy of which have a stronger affect to the xc energy.

Combining this fact with that of the previous subsection, we can conclude that the scaling method would provide correction preferentially for the certain parts of $\rho(\mathbf{r})$ and $n_{\mathrm{xc}}\left(\mathbf{r}, \mathbf{r}^{\prime}\right)$, which reduce the error $\Delta W$ effectively.

\section{CONCLUDING REMARKS}

In order to make the PD functional theory progress, two problems of the PD functional theory, i.e., the search region of PDs and approximate KE functional, should be addressed simultaneously. In this paper, we tackle two problems by means of the scaling method and approximate KE functional that are, respectively, described in Secs. II and III. Both the validity of the scaling method and the soundness of the approximate KE functional are successfully confirmed by actual calculations for the neutral $\mathrm{Ne}, \mathrm{Mg}$, and $\mathrm{Ar}$ atoms.
The striking feature of the scaling method is that it necessarily yields the corrected PD that satisfies the virial theorem rigorously. This is true so long as the KE functional is consistent with the exact relation Eq. (5) like Eq. (36). Due to this striking feature, the resultant electron-electron interaction energy, external potential energy, and $\mathrm{KE}$ are well balanced. The well-balanced correction leads to not only the drastic reductions of $\Delta W, \Delta V$, and $\Delta T$ but also the improvements of the electron density and xc hole. Especially concerning the latter, it is emphasized that the scaling method preferentially correct their spatial parts that affect the reductions of $\Delta V$ and $\Delta W$ more strongly. These improvements suggest that the corrected PD gets close to the ground-state one.

Furthermore, the scaling method does not need a heavy calculation additionally. This implies that the scaling method would be applicable not only to light atoms but also to heavier atoms.

\section{ACKNOWLEDGMENTS}

This work was partially supported by Grant-in-Aid for Scientific Research (Grants No. 22540390 and No. 23540446) of The Ministry of Education, Culture, Sports, Science, and Technology, Japan.
[1] P. Hohenberg and W. Kohn, Phys. Rev. 136, B864 (1964).

[2] W. Kohn and L. J. Sham, Phys. Rev. 140, A1133 (1965).

[3] U. von Barth and L. Hedin, J. Phys. C 5, 1629 (1972).

[4] M. M. Pant and A. K. Rajagopal, Solid State Commun. 10, 1157 (1972).

[5] G. Vignale and M. Rasolt, Phys. Rev. Lett. 59, 2360 (1987).

[6] G. Vignale and M. Rasolt, Phys. Rev. B 37, 10685 (1988).

[7] M. Higuchi and A. Hasegawa, J. Phys. Soc. Jpn. 66, 149 (1997).

[8] M. Higuchi and A. Hasegawa, J. Phys. Soc. Jpn. 67, 2037 (1998).

[9] M. Higuchi and K. Higuchi, Phys. Rev. B 69, 035113 (2004).

[10] K. Higuchi and M. Higuchi, Phys. Rev. B 69, 165118 (2004).

[11] K. Higuchi and M. Higuchi, Phys. Rev. B 71, 035116 (2005).

[12] Á. Nagy, S. Liu, and L. Bartolloti, J. Chem. Phys. 122, 134107 (2005).

[13] P. W. Ayers and P. Fuentealba, Phys. Rev. A 80, 032510 (2009).

[14] K. Higuchi and M. Higuchi, Phys. Rev. A 79, 022113 (2009).

[15] P. Ziesche, Phys. Lett. A 195, 213 (1994).

[16] P. Ziesche, Int. J. Quantum Chem. 60, 1361 (1996).

[17] A. Gonis, T. C. Schulthess, J. van Ek, and P. E. A. Turchi, Phys. Rev. Lett. 77, 2981 (1996).

[18] A. Gonis, T. C. Schulthess, P. E. A. Turchi, and J. van Ek, Phys. Rev. B 56, 9335 (1997).

[19] M. Levy and P. Ziesche, J. Chem. Phys. 115, 9110 (2001).

[20] F. Furche, Phys. Rev. A 70, 022514 (2004).

[21] Á. Nagy, Phys. Rev. A 66, 022505 (2002).

[22] Á. Nagy and C. Amovilli, J. Chem. Phys. 121, 6640 (2004).

[23] B. Hetényi, L. Brualla, and S. Fantoni, Phys. Rev. Lett. 93, 170202 (2004).

[24] J. K. Percus, J. Chem. Phys. 122, 234103 (2005).

[25] P. W. Ayers and M. Levy, J. Chem. Sci. 117, 507 (2005).

[26] Á. Nagy, Int. J. Quantum Chem. 106, 1043 (2006).
[27] P. W. Ayers, S. Golden, and M. Levy, J. Chem. Phys. 124, 054101 (2006).

[28] M. Higuchi and K. Higuchi, Physica B 387, 117 (2007).

[29] M. Higuchi and K. Higuchi, Phys. Rev. A 75, 042510 (2007).

[30] M. Higuchi and K. Higuchi, Phys. Rev. B 78, 125101 (2008).

[31] B. Hetényi and A. W. Hauser, Phys. Rev. B 77, 155110 (2008).

[32] K. Higuchi and M. Higuchi, J. Phys.: Condens. Matter 21, 064206 (2009).

[33] K. Higuchi and M. Higuchi, Phys. Rev. B 82, 155135 (2010).

[34] M. Higuchi and K. Higuchi, Phys. Rev. A 84, 044502 (2011).

[35] N. H. March and R. Santamaria, Int. J. Quantum Chem. 39, 585 (1991).

[36] P. W. Ayers, J. Math. Phys. 46, 062107 (2005).

[37] R. Cuevas-Saavedra and P. W. Ayers, Int. J. Quantum Chem. 109, 1699 (2009).

[38] D. Chakraborty and P. W. Ayers, J. Math. Chem. 49, 1810 (2011).

[39] K. Higuchi and M. Higuchi, Phys. Rev. A 85, 062508 (2012).

[40] R. G. Parr and W. Yang, in Density-Functional Theory of Atoms and Molecules (Oxford University Press, New York, 1989), Chap. 2.

[41] A. J. Coleman, Rev. Mod. Phys. 35, 668 (1963).

[42] Many-Electron Densities and Reduced Density Matrices, edited by J. Closlowski (Kluwer, Dordrecht/Plenum, New York, 2000).

[43] A. J. Coleman and V. I. Yukalov, Reduced Density Matrices: Coulson's Challenge (Springer-Verlag, Berlin, 2000).

[44] The Fundamentals of Electron Density, Density Matrix and Density Functional Theory in Atoms, Molecules and the Solid States, edited by N. I. Gidopoulos and S. Wilson (Kluwer, New York, 2003).

[45] E. R. Davidson, Chem. Phys. Lett. 246, 209 (1995).

[46] S. Kh. Samvelyan, Int. J. Quantum Chem. 65, 127 (1997). 
[47] M.-E. Pistol, Chem. Phys. Lett. 400, 548 (2004).

[48] P. W. Ayers and E. R. Davidson, Int. J. Quantum Chem. 106, 1487 (2006).

[49] P. Gori-Giorgi and A. Savin, Philos. Mag. 86, 2643 (2006).

[50] M.-E. Pistol, Chem. Phys. Lett. 417, 521 (2006).

[51] M.-E. Pistol, Chem. Phys. Lett. 422, 363 (2006).

[52] M.-E. Pistol, Chem. Phys. Lett. 431, 216 (2006).

[53] P. W. Ayers, Phys. Rev. A 74, 042502 (2006).

[54] P. W. Ayers and S. Liu, Phys. Rev. A 75, 022514 (2007).

[55] P. W. Ayers and E. R. Davidson, Adv. Chem. Phys. 134, 443 (2007).

[56] M.-E. Pistol, Chem. Phys. Lett. 449, 208 (2007).

[57] In this paper, we actually develop the KE functional that satisfies the exact relation Eq. (5), and apply it to the atomic structure calculations.

[58] M. Levy and J. P. Perdew, Phys. Rev. A 32, 2010 (1985).

[59] R. Courant and D. Hilbert, in Methods of Mathematical Physics Volume II (John Wiley \& Sons, New York, 1962), Chap. II.

[60] J. Wang and V. H. Smith, Jr., Phys. Rev. A 52, 1060 (1995).

[61] In Ref. [60], the nonrelativistic total energies of isoelectronic series of atomic ions that are estimated by combining experimental data and calculation data of the multireference single and double configuration interaction (MRSDCI) $[62,63]$ are employed in determining the coefficients of the $1 / Z$ expansion. By using the coefficients, the external potential and electron-electron interaction energies are calculated on the basis of the $1 / Z$ expansion method [60].

[62] S. J. Chakravorty, S. R. Gwaltney, E. R. Davidson, F. A. Parpia, and C. F. Fischer, Phys. Rev. A 47, 3649 (1993).

[63] E. R. Davidson, S. A. Hagstrom, S. J. Chakravorty, V. M. Umar, and C. F. Fischer, Phys. Rev. A 44, 7071 (1991).

[64] In the conventional DFT, the approximate xc energy functional has usually been adjusted correspondingly to the systems by using the parameters or by changing the functional forms employed. For example, in the LDA $+U$ method $[65,66]$, magnitudes of the parameters that describe the Coulomb interaction energy between the localized electrons are chosen correspondingly to the target systems.

[65] V. I. Anisimov, J. Zaanen, and O. K. Andersen, Phys. Rev. B 44, 943 (1991).

[66] A. I. Liechtenstein, V. I. Anisimov, and J. Zaanen, Phys. Rev. B 52, R5467 (1995).

[67] A. V. Bunge and R. O. Esquivel, Phys. Rev. A 34, 853 (1986).

[68] M. A. Buijse and E. J. Baerends, in Density Functional Theory of Molecules, Clusters, and Solids, edited by D. E. Ellis (Kluwer Academic Publishers, Dordrecht, 1995), p. 1. 